\title{
Communication in the Design Process of Web Animation Based on Scripting Language: Programmers' Perspectives
}

\author{
Jagan Mohan Balakrishnan ${ }^{1, \text { *, Soumya Manjunath Chavan }}{ }^{2}$ \\ ${ }^{1}$ Department of Humanities \& Social Sciences, Visual Arts, Jain University, Bangalore, India \\ ${ }^{2}$ Bengaluru School of Visual Arts, Karnataka Chitrakala Parishath, Bangalore, India
}

Email address:

jagan.multimedia@yahoo.com (J. M. Balakrishnan), soumyasaarc@gmail.com (S. M. Chavan)

${ }^{*}$ Corresponding author

\section{To cite this article:}

Jagan Mohan Balakrishnan, Soumya Manjunath Chavan. Communication in the Design Process of Web Animation Based on Scripting Language: Programmers' Perspectives. American Journal of Art and Design. Vol. 6, No. 2, 2021, pp. 38-46.

doi: 10.11648/j.ajad.20210602.11

Received: May 2, 2021; Accepted: May 18, 2021; Published: May 26, 2021

\begin{abstract}
Websites incorporate creative and complex web animations generated through scripting languages such as JavaScript. Programmers are responsible for implementing the web animations envisioned by the designers. The interdisciplinary communication between the designer and the programmer during the design process is a topic seldom studied. This qualitative research is based on an exploratory study primarily focused on the knowledge transfer and visualization of animation ideas. The study aimed to gather insights from professional programmers' experiences and understand their perspectives on web animation design based on scripting languages. The researcher conducted semi-structured interviews with a purposive sample of 12 programmers working with various organizations in South India. The individual interviews used open-ended questions to elicit in-depth information; it is a prevalent data-gathering method for the researchers to gather the participants' experiences. The researcher used the transcripts of the interview as the primary data for analysis. The results from the thematic analysis revealed imperative themes related to communication issues, the significance of the verbal and visual exchange of information, the new design challenges and the role of individuals in the overall design process. According to the programmers, the visuals are significant in knowledge transfer, and the team's communication gap has resulted from insufficient graphic representations. The study recommends improving the existing communication methods in web animation based on the scripting language. The study also revealed the significance of stakeholder's interaction during the design process and the multidisciplinary knowledge of individuals in improving interactions among the team.
\end{abstract}

Keywords: Design Process, Visualization, Team Communication, Web Animation, Visual Art

\section{Introduction}

The complex nature of design problems and the design process has been an area of study for many researchers. They develop efficient methods and better tools to support the design process that enhances the creative aspects and the quality of the final result [4]. Communication among the design team during the design is important because many individuals work together to achieve a goal. Communication among the individuals in a group is a critical factor, which decides the quality of output they deliver. The knowledge exchange during the design process involves both verbal and visual communication. Designers use different types of visuals extensively for communication. According to Simon, the designer's drawings act as an external memory aid during the design process [23]. "Knowledge Visualization" is a framework proposed by Burkhard, which describes the use of visual representations to improve the transfer and creation of knowledge between at least two persons [3].

An extensive literature is available on visualizations used by the designers to communicate design ideas to other individuals or team. Visualizations are external representations of an idea, it is the designed visual information that designers want to convey, and these 
designed visuals are the most desirable way of communication for them. The type of visual representations and medium depends on the domain and projects. The designers follow different representation techniques based on the domain, requirement and the messages that need to be conveyed. There are conventional methods of visual representations, such as simple jottings by the designers. Drawings are an integral part of most of the design activity; It is a 'Thinking tool' for designers $[8,13,26]$ and one of the fundamental and original ways of communication. The visualization needs of the recent design disciplines such as digital media and animation are hefty. The design processes have evolved due to the complexity of the problems the designers have been engaging. The designers are looking for newer means of visual representations since the communication of ideas among the team is essential for the success of these projects.

Effective communication among team members is an area that needs to be addressed adequately. Cronin and Weingart identify inconsistencies in shared knowledge between individuals in the design team and call it representational gap; it defines one of the team's problems [6]. It is about the uneven transfer of relevant information among team members in the context of design activity. Representational gaps are a process loss phenomenon [25] which will affect the overall coordination and cause a quality loss in the design process. Identifying the nature of the representational gap in the given context and the deeper understanding may reduce the conflicts they create [5]. The knowledge transfer among the team is significant; since each member of the team should understand other members' information needs [4].

This study explores the themes related to information exchange and visualization design in web animation design. The current web industry uses scripting language for generating web animation- an animation that uses the programming language to create sophisticated and creative visuals and animation. The script-based web animations seem to be the future with the introduction of $\mathrm{Html} 5$, the latest web standard. For example, The JavaScript $3 \mathrm{D}^{1}$ libraries, such as three js, provide the opportunity to build script-based animations. Three js is JavaScript Library used to create 3D computer graphics based on WebGL, a JavaSript Application Programming Interface to render computer graphics in a web browser. The website www.threejs.org provides valuable insights and references for script-based web animations [29]. Figure 1 illustrates an image sequence from a JavaScript-based animation rendered using WebGL. The following four frames present a 3D geometry called "TorusKnot Geometry" and its rotation along its Y-axis.

The visualization of innovative design ideas before the final production is significant in many creative and artistic disciplines, such as films and 3D animation movies. While observing the new age web animations, it is definite to think about the visualization technique used during the design process for prototyping animation. This context invokes a

${ }^{1}$ Three Dimensional relevant question, "How do the designers communicate animation ideas of script-based web animation to programmers?". The background study through literature and the interviews with the stakeholders of web animation reveal that the transfer of ideas includes conventional communication methods such as verbal communication and visualizations such as drawings, images, wireframes, storyboards, and 2D animation. The medium of the web and the technologies associated with it are continuously evolving, which creates new challenges to the design world. The websites consist of vibrant visuals that present interactive and animated content. The use of traditional animation on websites is obsolete now because of the introduction of script-based web animation. The domain is looking for a newer way of expression of the content. The changing technologies are adding more dynamism to the visual medium.

The visualization in a design process majorly discusses the communication of ideas through visual designs. The general perception is that the designers are creativity oriented while programmers are more technically oriented. It is necessary to understand how the programmers perceive, understand, and execute the ideas created and conveyed through different communication means by the designers. The communication among the team members includes discussion through visual artefacts, including animation, to represent ideas. It is interesting to understand the interaction between the designer and the programmer on delivering design ideas and their visual presentation, also the programmer's perception regarding the merits and demerits of different communication channels used in the design process.

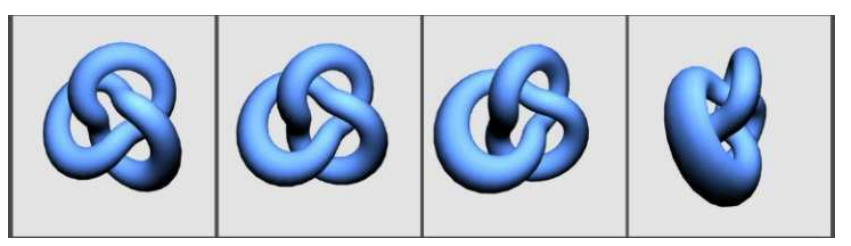

Figure 1. Frames from JavaScript-based web animation.

\section{Programmers' Perspectives on the Design Process}

Web design and animation are a collaborative effort of design teams consisting of individuals from different disciplines such as graphic design, animation, and programming. The study aimed to gather insights from professional programmers' experiences and to understand their perspectives on communication and visualization of web animation based on scripting languages.

\subsection{Research Design}

This qualitative research aims to study the subjective experiences of programmers who create web animations based on the designer's ideas and creative brief. The inquiry strategy is based on phenomenography [17] [3030], a 
data-gathering method where the participants describe their experiences. The study involves semi-structured interviews [11] of the programmers. The individual interviews help to elicit in-depth information regarding communication among team members in script-based web animation design. The primary focus of the one-on-one interaction is on the matters in communication through visualization. This study uses an inductive approach in the analysis of data. Thematic analysis will be used to analyze the transcripts from the semi-structured interview; the analysis is grounded on the data; the resulting themes and inferences will be reported.

\subsection{Data Collection}

The study uses semi-structured interview to collect primary data from individual programmers working with various organizations. The diversity of the sample population achieved by selecting participants from different firms. The firms situated in multiple locations in south India. The participants signed a consent form for their total participation in the study. Interview dates scheduled as per the convenience of the participant. Most of the interviews were face-to-face. Some of them were ready to attend the meeting via phone calls and other communication channels such as email and mobile applications such as WhatsApp. The interviews conducted as per schedule with audio recording and prepared transcripts. Few of the participants were voluntary, while others received a token of appreciation for their kind gesture. A total of 12 interviews conducted, and it took a minimum of 45 minutes to a maximum of 90 minutes to complete each interview.

\subsection{Participants}

The education and experience in the respective domain are the selection criteria for the participants. The researcher used purposive sampling to identify participants for the interview. Data saturation is significant in both sample size estimation and qualitative research design. Literature suggests a sample size of 12 is sufficient to gather qualitative data from in-depth interviews. [15]. Out of 12 interviewees, 11 people are graduates. Two participants are post-graduates. The participants are well informed and have sound technical skill-sets. It is a notion that the well-experienced participants will have a better understanding, but we also included the novice programmers in the study for maximum variation. The following table (Table 1) presents the details of the participants and their experience.

\subsection{Semi-structured Interview}

The interview uses open-ended questions to elicit in-depth information about the web animation design process and the communication aspects. The formation of interview questions requires following the guidelines of qualitative research methodology. The researcher's rapport with the participants is crucial in a semi-structured interview [11].

\subsection{Questionnaire}

The questionnaire involved nine questions. The open-ended questions expect a more in-depth and broader response; hence, the resulting information will be comprehensive. The transcription of recoded audio is the primary data corpus for the coding and analysis of the study.

Table 1. Experience details of the participants.

\begin{tabular}{ll}
\hline Interviewee Code & Experience (in Years) \\
\hline P1 & 8 \\
P2 & 8 \\
P3 & 10 \\
P4 & 10 \\
P5 & 5 \\
P6 & 2 \\
P7 & 3 \\
P8 & 7 \\
P9 & 2.5 \\
P10 & 2 \\
P11 & 2 \\
P12 & 15 \\
Average Experience & 6.2 Years \\
\hline
\end{tabular}

\subsection{Thematic Analysis}

The transcripts from the interview require a detailed and exhaustive examination, namely, thematic analysis. It is an analysis that involves "identifying, analyzing, and reporting patterns within data" [2]. The researcher examined the transcript to identify the meaningful chunks called codes through line-by-line scanning. The patterns of shared meaning across coded items called a theme; it is originating from the code. The focus of the whole process is to identify meaningful data and interpret meaning. Figure 2 represents the fundamental process of the analysis.

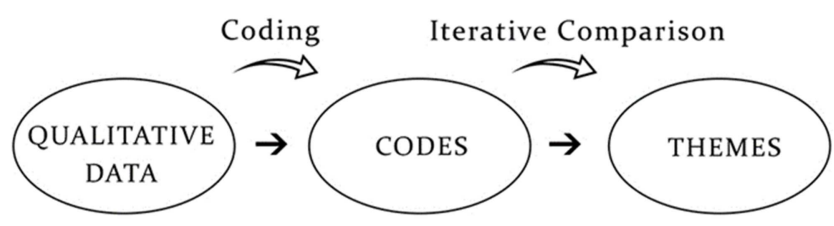

Figure 2. Thematic Analysis Process.

There are different qualitative investigation techniques for analyzing extensive data in transcripts; each of these analyses is thematic analysis. It is a common practice in humanities and social science. The data collection through in-depth interviews provides data on human experience, mainly in a narrative form. The researchers make sense of the transcript through careful reading; it helps them familiarize the content. The researcher uses his knowledge, reasoning, and observations to make sense of the material. He analyses the data extracts from the participants' diverse opinions and identifies and reports potential themes and meanings.

\section{Results}

The open-ended questions explored the overall design process, the communication and the information design during the design process, visual communication and recent design process trends. 


\subsection{Programmers' Views}

This section reports the perspectives of programmers on various aspects of the design process.

The challenges in the design process: The Programmers assuming communication issues in the design process at various levels. According to them, poor communication disrupts the harmony among the team, the overall workflow, and ultimately the end product and the user. The efficiency of communication affected by the way they express ideas, mediums used by the team. The knowledge transfer among the group affected by the communication skill of the individuals and the visuals used by them. The programmers perceived that the visuals currently using in the design process are not sufficient in communicating design ideas of web animation

The factors that affect communication: The communication between the designer and the programmer might disrupt due to the difficulty in conveying creative design ideas. Creative ideas are hard to communicate. The conventional visualization techniques such as drawings lack many qualities to present the dynamism of recent web animation. According to the programmers, the efficiency of visualization is significant. The traditional media often fails to convey the exact ideas. Also, technical factors are not easy to convey. The conventional way of communication not delivering the technical aspects of digital media. The professional performance of individuals includes his communication, coordination, and co-operation smatters. The professional experience of the individual and their limitations may affect the communication.

Designers' communication strategies: According to the programmers, effective communication is possible through appropriate visualizations and regular meetings. The verbal explanation during the consultations can convey a lot, including emotions. The visual artefacts such as images, drawings, PPT, animations can support the knowledge transfer.

Use of Visuals in communicating animation ideas: All the Participants are expressed that the visuals used during the design process are significant in the communication. The visuals, including animations used by the designers, can present design elements, composition, timing, motion, duration, minor details and an overview of the project.

Techniques used in communicating animation ideas: Programmers are more comfortable with visuals. The designers use various methods to express ideas it includes Verbal communication, Documentation, Wireframes, Drawings, Images from graphics design tools, Storyboard, Animation, PowerPoint presentation, Video, Reference Websites \& Templates and Prototypes for communicating animation ideas.

The best way to communicate animation ideas: The best communication method is subjective and based on personal preference. According to programmers, the best practices of communication is using visuals and visual prototypes and creating prototypes using software like Aftereffects, Adobe Flash. The other communication channels include Face to face communication, storyboards, sketches, website references, PowerPoint presentations and videos.

Effective of current communication: The programmers are adapted to the current visualization techniques, but better methods must be tried. According to programmers, they felt the communication is not sufficient; sometimes, the clients failed to communicate their ideas effectively.

Programmers 'perception of the effectiveness of the current communication: The programmers' perception is that the $2 \mathrm{D}$ images used for the information exchange are insufficient for the knowledge transfer. Good references can convey ideas, which is essential for client approval. The references are always better than verbal communication.

Views on Improving communication: Communication can be improved by using more effective visualizations, incorporating better communication methods, and using proper references. Most of the participant emphasized the importance of reference and the overall team effort required to produce sound output. Increase the interactions with stakeholders, understand others, including their emotions, through close monitoring of the project and process.

\subsection{Themes from the Study}

The transcribed data from the interviews were organized in Microsoft Word for coding. The investigation uses transcribed data from the interview to explore the views of programmers and reports narratively. The themes are abstracted from the coded data organized into Basic Theme, Organizing Theme, and Global Theme. The lowest order theme from the data being 'Basic theme,' followed by a middle-order theme called 'Organizing theme' and the higher-order 'Global Theme,' which encompasses a broader idea. [1]. Table 2 presents the summary of themes that emerged from the investigation.

Table 2. Summary of themes.

\begin{tabular}{lll}
\hline Basic Themes & Organizing Themes & Global Themes \\
\hline $\begin{array}{l}\text { Animation } \\
\text { Design Iteration }\end{array}$ & Visual Representation & Visual Design \\
$\begin{array}{l}\text { Repeating Designs } \\
\text { Communication Method }\end{array}$ & Creativity & \\
$\begin{array}{l}\text { Teamwork } \\
\text { Time management }\end{array}$ & Collaboration & \\
$\begin{array}{l}\text { Professional Experience } \\
\text { Client satisfaction }\end{array}$ & Participatory Design & General \\
$\begin{array}{l}\text { Knowledge Transfer } \\
\text { Knowledge Disparity }\end{array}$ & & \\
Interdisciplinary knowledge & Bridging & \\
\hline
\end{tabular}

\subsubsection{The Basic Themes}

This section presents the basic ideas expressed by the programmers.

Animation: The web animation presents many design elements such as shapes, colour, texture, composition, dimensions, depth, space, movement, interaction, visual style, and emotions. The communication of motion is the most complicated process. Designers communicate the design ideas through different visual representations and animation with the help of recent technology.

Design Iteration: Designers happen to create many visual 
prototypes before the final product. The final design is an output of many iterations. The lack of communication among individuals or teams leads to many iterations.

Design Repetition: Many design ideas are repeatedly using in web animation because clients and designers often use similar references. An appealing new design from the web will create a new trend, and it will continuously reflect or repeat in other projects. Several users adopt it and frequently use it as a reference for their project, such repetition considered a lack of creativity.

Communication Methods: Verbal communication is the primary method of idea transfer in the Design Process. It helps in understanding the essential requirement, including the emotional aspects, by elaborating needs and explanations. Though it is a less desirable medium for designers, many stakeholders expressed the effectiveness of animation and other visual communication methods. Visual aids are considered one of the best tools for collaboration because they can communicate directly without language barriers.

Teamwork: This is one of the critical factors in achieving the expected result in the design process. It is possible only through proper communication among individuals. So, each member should possess professionalism based on the attitude and knowledge to understand the design problems that other members face. The members of the team expected to work as a coherent group to achieve a common goal.

Time management: It is a significant factor, but efficiency disturbs due to various reasons such as communication. Many participants have shown concerns about time management, and it is always desirable for any stakeholder to complete the project on time.

Professional Experience: The design process involves managing different design problems. It is a common notion that experienced designers have a better understanding of the issues. The opinions of the participants emphasized this assumption.

Client satisfaction: The theme is repeated many times in the conversations. It is desirable to achieve the goal with maximum accuracy, which is the desired output visualized by the client. The client primarily communicates to a marketing executive, designer, or programmer. The client must have an idea about the outcome, and he must be consistent during the design process. Many of the programmers complain about the inconsistency of the client on his decisions and requirements in the overall design idea. If the design concept or, more precisely, the animation concept is not clear initially, it takes extra time and effort to fix the final design.

Knowledge Transfer: The team members' communication and effective transfer of design information are critical in achieving the result and the team's overall success. The whole exercise is to accomplish a common goal, so it is a best practice to mention, visualize the overall project, and the individual responsibilities in advance. Knowledge transfer is possible with proper communication and visual aids.

Knowledge Disparity: The design as a discipline, the stakeholders, possesses a different level of knowledge and awareness about its design, principles, and associated technologies. Such a situation leads to miscommunication among the team. So, individuals should equip with multidisciplinary expertise. Individuals' knowledge differs according to education and experience. Web animation's design process is not complete without technical aspects, such as the medium of display, the system's processing power, screen size, and resolution.

Interdisciplinary knowledge: Different ways of communication methods exist for idea transfer and communication among team members. The multidisciplinary expertise that each member is possessing will enable them to understand others' views better.

\subsubsection{The Organizing Themes}

This section presents the secondary level of ideas formed from the primary themes.

Visual Representation: The data revealed many aspects of visual communication in web animation's complex design process and the importance of visual representations in supporting script-based web animations. In the discussion, participants expressed their views on the challenges a team faces while executing the design. The understanding of client requirements and their satisfaction is the most critical aspect during the design process. Verbal communication is not adequate to understand the design concepts, especially some design ideas that involve motion or animation. The programmers feel a lack of clarity in the communication of animation ideas due to insufficient visual representation. Programmers expect more precision in visual communication and proper illustration of ideas through complementary visualizations to understand the actual requirement and emotions to complete a project within the time frame.

Creativity: The designers use visual representations to externalize their ideas to see, enhance, and communicate creative design ideas to others. There is a range of visual communication methods used in the design process. Verbal communication with a combination of conventional visual communication methods can explain ideas more effectively. In the context of communicating animations ideas in script-based web animation, most of them expressed reference websites as a first preference; it facilitates the ease of understanding. The lack of appropriate reference will be a challenge for the programmer to understand an original creative idea. Hence, the creative ideas for the designs must be presented visually with complementary visualization mediums.

Collaboration: Many aspects lead to miscommunication, such as limitations of conventional representation mediums, technical aspects of the medium, and inexperience of stakeholders. Early detection of the exact requirement will save time and effort. It is preferable to improve communication through a combination of verbal communication, documentation, and conventional visualizations such as images, storyboards, animations, ppt presentations, and prototypes created using 2D animation software. Despite the use of all these communication methods, most of the programmers pointed out that several iterations are 
required to finish the project, which in most cases involves an extension of the deadline because of unclear input from the designer or client. Coordination and co-operation are the two ideas that emerged in the current context. As per the participants, the collective effort of verbal communication, visual representations, and references help in achieving true collaboration.

Participatory Design: The active involvement of all the stakeholders is expected in the design process. Such a process and procedures create a desired and good result. The designs can be very creative hence intricate, less reasonable. At the same time, from the production point of view, the output must be achievable within the project's scope. The designer and programmer's agreement is critical in delivering the design results in an ideal manner. The programmer's perception is that a harmonious environment will create a better team collaboration. Every project has a limit on its available resources. Many factors affect the successful completion of a project, such as total cost and time, experience, and programmers' knowledge. The programmer expects a consensus on the final design, and they prefer a creative and productive environment. The team should reach a consensus on the feasible plan within the context. The result must be achievable, and ultimately it should satisfy the client's expectations.

Bridging: Team members always expect fair sharing of knowledge during the design process. Each team member has a different knowledge base. The understanding of each other's views is essential in decision making. Client, designer, and programmer have their knowledge and perspectives. It suggests that all the stakeholders, part of the design activity, must understand other domains involved in the overall design, which makes a significant difference in total communication among the team. Some programmers suggest a proper discussion among the group before finalizing and committing any design ideas to the client because sometimes the designer might not be familiar with the recent technologies the programmer is dealing with, which may create conflicts among them. It is observed that, in some situations, especially in smaller organizations, the role of a designer and a developer is overlapping. In some cases, the client discusses the requirements directly with a programmer.

\subsubsection{Global Themes}

Two global themes represent the broader ideas conversed in the interview; visual design and general interaction.

Visual Design: The overall data provides a broader idea of information design and visualization in the design process. An external representation of the plan simplifies communication. It gives a visual reference for the stakeholders and helps in developing creative ideas.

General Interaction: The interaction among the stakeholders of the design touches on different aspects of communication. A successful interaction encompasses various aspects of collaboration, bridging, and participatory design. Meaningful communication enhances teamwork among professionals; ultimately, it helps in effective knowledge transfer and better results. The interaction can significantly improve when the professionals possess experience and multidisciplinary knowledge.

\section{Discussion}

Visual Design: The programmers advocate that visualizations help in communication; it aligns with the idea presented by Schon that visualizations provide visual cues for creative ideas [21], thus significant in communicating creative ideas. The technical knowledge and individual professional qualities affect the overall process of communication.

Designers create step by step instruction through visuals and visual prototypes. Such artefacts are important as a resource for communication [20].

The programmers' views of visuals are in line with the thought about "Boundary objects", the artefacts used in the design process, which acts as a mediator among stakeholders [24]. They contribute to different communication levels of details. According to the programmers, it is a communication method that acts as a reference for them. The visuals are providing details to an overview of the entire design.

The visual prototypes can provide a reference for many aspects such as graphic style, the uniqueness in its appearance and creation method [9] and motion, the depiction of movement. This idea aligns with Vosniadous' view on analogical reasoning and resemblance; the final design and the prototype are similar in some respects [31].

The significance of visual in convey messages are unquestionable; the visual messages are significant [18]. Visuals are substantial in education [16], and visuals are essential in the design process [13].

Most of the programmers prefer visual communication rather than verbal. The current communication methods include conventional methods such as drawings. Some of the participants were touched on the $3 \mathrm{D}$ animation as a visualization technique. Visualizations are guiding the design process by providing feedback on the design ideas [22]. The 2D drawings are not fully useful for conveying animation. They suggest that promising prototypes created using animation can be utilized for more significant projects.

General Interactions: According to the programmers, the most significant challenge is the communication of ideas, Effective communication is significant in any design process, but there are situations, messages are not delivered or understood adequately; researchers call it a "representation gap" [6]. A representation gap occurs because of the different knowledge levels and values that each individual possesses. These knowledge gaps can even happen in what appears to be homogeneous teams [28]. The representation gaps create conflicts among team members. The design team is working together to solve a given problem. However, these conflicts intensify when the team has individuals with different backgrounds and understandings about the given situation.

The programmers recommended refining conventional methods of communication. The visualizations should support by verbal instructions. The face-to-face communication can be 
recorded so that the content can be revised later. The interaction doesn't create any new ideas for the significant improvement of communication. All the participants discussed conventional methods and emphasized general techniques; there are no recommendations for improving visualizations but increase the uses of visualization and references. More interactions with stakeholders, using verbal and visual methods. Use of enhanced communication methods like visualizations and references. At the same time, the efforts of individual professionals in a project, team effort, and stakeholder meeting are required to understand the requirements. The overall knowledge transfer process is possible when an individual has empathy as prescribed by Rifkin [19] and design co-operation suggested by Eckert [7].

The two major ideas discussed are Visual design and general interactions. The thematic segregation of the inferences primarily points to the visual design part, and the following points summarize the visual design aspects in the current context.

1. Programmers believe that visuals are an ideal and preferable way of communication.

2. Programmers use various visual representations and techniques such as drawing, images, wireframe, and storyboard for information design. The programmers prefer a visualization that complements the final design.

3. The current communication channels are helpful, but they can be enhanced by appending or changing with the corresponding animation based on 2D and 3D animation.

4. The programmers suggest animation for information design and similar visual references in understanding the design elements and representing motion

5. Visualizations help in exploring creative ideas.

The views reveal the problematic nature of information design in the design process. The programmers explicitly expressed the lack of adequate visual information. The analysis of participants' views clearly shows that the team's communication gap resulted from insufficient graphic details.

The visuals are not the sole focus of the conversation; the other opinions on general interactions in the design process matters. The following points summarize the general interaction aspects in the current context.

1. Multidisciplinary knowledge of team members can improve communication, coordination, and collaboration.

2. The lack of interactions prompts an increase in design iterations, which affects the design process inversely.

3. The proper sharing of knowledge is an essential aspect of the design process. It reduces the knowledge difference through enhancing the knowledge transfer

4. To meet the client's expectation, a designer should deliver the final design visualized by the customer within the scheduled time.

The researcher identified certain circumstances in the design process, which affects the overall design activity. The client's communication skills, designers' understanding of clients' needs, the designer's visualization skills, and finally, the programmer's knowledge of the requirements also contribute to the interaction. The design process's flow may affect the clients' deviation from the original plan or idea in the later stage of the process, which causes excess time and resources to complete the project. The programmer's opinion is vital in the final stages of the design process before finalizing the animation design

The team's interaction must have clarity that will help better understand the team, leading to a collective agreement on the final output. The programmers often feel a lack of understanding and conflicts among the design team while reaching a consensus.

In a design process, the message's clarity is most significant to reduce conflicts and miscommunication among members. A precise exchange of information using a suitable medium helps reduce the number of iterations of the design, thus helping in meaningful collaboration and agreement on the final output. So, designers prefer visual communication through visual artefacts in design projects. The existing ways of communication are helpful and practical, but more effective methods are preferable. As Goel's account visualization helps them to achieve preferred excellence in the result [13].

Programmers expressed that the current visualization techniques are not adequate for conveying animation ideas, which leads to many design iterations to finalize a project. The ideas can be expressed clearly through visuals, so the design iterations can be minimized. The information design based on corresponding visual models is preferable to communicate ideas of script-based web animation. The study also reveals the importance of interdisciplinary knowledge among team members. In a human-centered design context, understanding others' needs is essential. According to Firth, the base of each team members' perspectives is directly associated with their knowledge, experience, and role in the organizational system [10]. The study suggests that sharing of knowledge, updating knowledge, and understanding each other's difficulties are significant in solving design problems. The overall concepts are in alignment with Gardner's idea of team integration; according to him the team integration is possible only through a careful, systematic process, and it must be efficient, collaborative, and [12].

\section{Conclusion}

The team should build a shared mental model to understanding the design ideas. The visual representations help in creating such a mental model. Lack of understanding among the team may create conflict. Design being a social activity, communication through visuals helps individuals to understand others' views.

The design process of programming-based animation follows a complex design process. The inferences from the study show a communication gap among the designers and programmers due to insufficient visual design. Participants emphasize the shortage of interdisciplinary knowledge among the stakeholders. Multidisciplinary familiarity is significant in teamwork. In such situations, proper information visualization is beneficial. The excess number of iterations required to 
complete the project might be a pointer towards the existing communication process's inability and precision. Proper information design through appropriate visualization can efficiently influence the design process and reduce unnecessary iterations, total time requirement, and resources. Participants are confident about the information transfer through visual representations. They prefer animation from reference websites as a way to communicate animations ideas. It suggests that a complementary visual style and reference is preferable to deliver an animation idea. Without appropriate visual information, referred to as visualization, it is hard to convey and to convince a creative plan or idea to an individual or a team. Thus, it is evident that new ways of representations are desirable to express innovative designs.

\section{Recommendations}

Animation based on programming is a complex design scenario with broader implications in interactive and immersive web [27]. The communication of design ideas in the context of web animation needs to be addressed with appropriate visualization. Digital technologies provide computer graphics-based visualizations such as previsualization [14], a visualization technique based on 3D animation; which could sufficiently communicate web animation ideas. Such visual representations may help to build shared mental models in design teams; further studies are required to verify the usefulness of similar communication techniques.

\section{Acknowledgements}

I thank the individual programmers who provided time for the interview and participated with great enthusiasm. I want to thank my guide Dr Soumya Manjunath Chavan for valuable advice.

\section{References}

[1] Attride-Stirling, J. (2001). Thematic networks: an analytic tool for qualitative research. Qualitative research, 1 (3), 385-405.

[2] Braun, V., \& Clarke, V. (2006). Using thematic analysis in psychology. Qualitative research in psychology, 3 (2), 77-101.

[3] Burkhard, R. A. (2005). Knowledge visualization: The use of complementary visual representations for the transfer of knowledge. A model, a framework, and four new approaches (Doctoral dissertation, ETH Zurich).

[4] Clarkson, J., \& Eckert, C. (Eds.). (2010). Design process improvement: a review of current practice.

[5] Cronin, M. A., \& Weingart, L. R. (2005). Conflict in diverse teams: The problem of representational gaps and the solution of cognitive and affective integration. 1-45.

[6] Cronin, M. A., \& Weingart, L. R. (2007). Representational gaps, information processing, and conflict in functionally diverse teams. Academy of management review, 32 (3), 761-773.
[7] Eckert, C., \& Boujut, J. F. (2003). The role of objects in design co-operation: communication through physical or virtual objects. Computer Supported Cooperative Work, 12 (2), 145-151.

[8] Ferguson, E. S. (1992). Engineering and the Mind's Eye. MIT press.

[9] Fernie, E. (Ed.). (1995). Art history and its methods: A critical anthology (p. 361). London: Phaidon.

[10] Firth, B. M., Hollenbeck, J. R., Miles, J. E., Ilgen, D. R., \& Barnes, C. M. (2015). Same page, different books: Extending representational gaps theory to enhance performance in multiteam systems. Academy of Management Journal, 58 (3), 813-835.

[11] Flick, U. (2009). An introduction to qualitative research. sage.

[12] Gardner, H. K., Gino, F., \& Staats, B. R. (2012). Dynamically integrating knowledge in teams: Transforming resources into performance. Academy of Management Journal, 55 (4), 998-1022.

[13] Goel, V. (1995). Sketches of thought. MIT Press.

[14] Jaganmohan, B and Chavan, S. M. (2017) Previsualization as a Tool for Visualization: A Thematic Analysis of Experts' Perspectives. AGU International Journal of Research in Social Sciences \& Humanities, Vol. 5, 304-312.

[15] Mason, M. (2010, August). Sample size and saturation in $\mathrm{PhD}$ studies using qualitative interviews. In Forum: qualitative social research (Vol. 11, No. 3).

[16] Mayer, R. E. (1994). Visual aids to knowledge construction: Building mental representations from pictures and words. Comprehension of Graphics, In Advances in psychology (Vol. 108, pp. 125-138). North-Holland.

[17] Orgill, M. (2012). Phenomenography. Encyclopedia of the Sciences of Learning. US. 2608-11.

[18] Pettersson, R. (2002). Information design: An introduction (Vol. 3). John Benjamins Publishing.

[19] Rifkin, G (1994) 'Product development: empathic design helps understand users better' Harvard Business Review Vol 72 No 2 pp 10-11.

[20] Robertson, T. (1996). Embodied actions in time and place: The cooperative design of a multimedia, educational computer game. Computer Supported Cooperative Work (CSCW), 5 (4), 341-367.

[21] Schon, D. A. (1983). The Reflective Practitioner: How Professionals Think in Action. London.

[22] Seitamaa-Hakkarainen, P., \& Hakkarainen, K. (2000). Visualization and sketching in the design process. The Design Journal, 3 (1), 3-14.

[23] Simon, H. A. (1981). The sciences of the artificial. MIT press.

[24] Star, S. L. (1989). The structure of ill-structured solutions: Boundary objects and heterogeneous distributed problem solving. In Distributed artificial intelligence (pp. 37-54). Morgan Kaufmann.

[25] Steiner, Ivan Dale. (1972). Group process and productivity. Academic press. 393-422. 
[26] Suwa, M., \& Tversky, B. (1997). What do architects and students perceive in their design sketches? A protocol analysis. Design studies, 18 (4), 385-403.

[27] Tacgin, Z. (2020). Virtual and Augmented Reality: An Educational Handbook. Cambridge Scholars Publishing.

[28] Tetlock, P. E. (2000). Cognitive biases and organizational correctives: Do both disease and cure depend on the politics of the beholder? Administrative Science Quarterly, 45 (2), 293-326.
[29] Threejs. Org. (2018) Threejs. Org, https://threejs.org. Accessed 9 Jan 2020 .

[30] Tight, M. (2016). Phenomenography: The development and application of an innovative research design in higher education research. International Journal of Social Research Methodology, 19 (3), 319-338.

[31] Vosniadou, Stella, and Andrew Ortony, eds. (1989). Similarity and analogical reasoning. Cambridge University Press. 